\title{
1 No evidence that male sexual experience increases mating success in a
}

\section{2 coercive mating system}

3 Maider Iglesias-Carrasco ${ }^{1 \dagger}$, Rebecca J Fox ${ }^{1 * \dagger}$, Alan Vincent ${ }^{1}$, Megan L. Head ${ }^{1}$, Michael

$4 \quad$ D. Jennions ${ }^{1}$

5

$6{ }^{1}$ Division of Ecology and Evolution, Research School of Biology, Australian National

7 University, Canberra, ACT 2601, Australia

8

9 †These authors contributed equally to this work

10

* Correspondence: R. Fox, Division of Ecology and Evolution, Australian National

University, Canberra, ACT 2601, Australia

E-mail address. rebecca.fox@anu.edu.au

T: +612 61254947

Abstract:

Several studies have shown that sexual experience can alter a male's mating behaviour to increase his future mating success. One explanation is that experienced males are better at courting females and inducing them to mate. Experienced males might also be better at identifying higher quality mates, although fewer studies have tested for this benefit. In both cases, however, these potential benefits of sexual experience might be partially offset by the energetic costs of courting and mating, which tend to reduce a male's subsequent ability to invest in sexually selected traits, and thereby reduce his future attractiveness and mating success (i.e. hasten the onset of reproductive 
senescence). Here we use the eastern mosquitofish, Gambusia holbrooki, to test whether

27 sexual experience elevates male mating success. We housed recently-matured males either with full mating access to females (experienced males), or in the visual and olfactory presence of females with whom they could not mate (naïve males). We then measured: the strength of male mate choice for larger, more fecund, females; male mating behaviour (time spent chasing females and the number of copulation attempts) and insemination success. Experienced and naïve males did not differ significantly in their mating behaviour and there was no effect of sexual experience on the likelihood of mating, or on the number of sperm inseminated (although experienced males had a tendency to be less successful when performing gonopodial thrusts). Experienced males in two-choice trials were, however, significantly more likely to "inspect" both females,

37 and had a significantly stronger preference for larger females. Finally, we measured male immune response and growth to test for any costs of the increased mating effort that is concomitant with greater experience. Experienced males had significant slower post-maturation growth and a significantly weaker immune response than naïve males.

42 Keywords: life-history trade-offs, male mating success, Poecillidae, sexual selection 


\section{Introduction}

4

In many species a male's ability to acquire mates depends on sexually selected morphological and behavioural traits that increase his attractiveness or ability to repel rivals (Kuijper, Pen, \& Weissing, 2012). These costly sexual traits are often expressed in a context-dependent fashion in response to variation in factors such as food availability (Bonduriansky, 2007), the level of mating competition (Bretman, Gage, \& Chapman, 2011), the intensity of sperm competition (Fitzpatrick \& Lüpold, 2014; Kelly \& Jennions, 2011), and the risk of predation (Kotiaho, 2001). In addition, it is possible that males alter their investment in sexual traits in response to experience gained during sexual encounters. For instance, it has been suggested that as males interact with females they learn to be more effective at seducing or coercing females into mating (e.g. Dukas 2005; Dukas et al. 2006; Pérez-Staples et al. 2010). However, relatively few studies are designed to distinguish between learning and other factors (e.g. female preferences for older males, or direct effects of male age) that might increase the mating success of more sexually experienced males.

To date, research on how sexual encounters influence subsequent male reproductive success has tended to focus on female mate choice for more experienced males (Edvardsson et al. 2008; King and Fischer, 2010). These studies often show that females prefer older, potentially more experienced, males (review: Brooks and Kemp 2001). However, few studies have explored how past sexual experience influences a male's subsequent sexual behaviour and his ability to aquire mates and successfully copulate, independent of changes in female mating preferences that depend on his mating history (but see Saleem et al. 2014; Balaban-Feld and Valone, 2017). 
During sexual encounters with females, males can learn to adjust behaviors such as courtship to increase their likelihood of mating (e.g. Dukas et al. 2006; Pérez-Staples et al. 2010). Similarly, in species where sexual coercion is the dominant male mating tactic, males with previous exposure to females might learn tactics that make them more successful at sneak copulations than sexually naïve males. For example, in mosquitofish juvenile males reared in the absence of females made significantly more copulatory attempts as adults than did those reared with females (Bisazza et al. 1996). However, this study did not quantify the potential benefits of adult male sexual experience due to improvements in mating tactics. In addition to learning how to adjust mating behaviour to increase reproductive success, sexual experience might allow males to better assess mate quality (review: Verzijden et al. 2012). Learning can happen through sexual imprinting early in life (Immelmann, 1975), but it can also occur when mature individuals gain experience with potential mates. For example, male damselflies learn to discriminate between conspecific and heterospecific females based on previous courtship interactions (Svensson et al. 2010). Similarly, in damselfly species with discrete female morphs, male prefer the female phenotype with which they have previously had a successful mating (Fincke, Fargevieille, \& Schultz, 2007). In fruitflies, males that have previously interacted with unreceptive, recently mated females spend less time than inexperienced males courting such females in future encounters (Dukas, 2005). In sum, mating preferences are often plastic, with individuals adjusting their mate-choice decisions based on their social environment (e.g. Mautz and Jennions 2011; Gasparini et al. 2013; Macario et al. 2017; review of male maet in choice poecilid fish: Schlupp 2018) and past encounters with mates (e.g. Reif et al. 2002; Balaban-Feld and Valone, 2017). 

increase in mating effort. Mating effort, such as investment in pre- and post-copulatory sexual traits, or even the cost of learning itself (Mery \& Kawecki, 2003), can reduce the availability of resources needed for self-maintenance. Mating behaviours, like courtship or harassment of females, can impose costs on males that include increased energy expenditure and loss of time for other activities such as foraging or evading predators (Kelly \& Godin, 2001; Kolluru \& Grether, 2005). Similarly, investment in ejaculates trades off with other fitness-enhancing traits and reduces the resources available for survival, precopulatory sexual traits and general maintenance (Barnes \& Partridge, 2004). The energetic costs of mating that are associated with gaining sexual experience might therefore interact with age to elevate male reproductive senescence. Prolonged sexual activity has been related to reduced male longevity, a decline in body mass, and weaker immune response in many species (e.g. Olsson et al. 1997; Rolff and Siva-Jothy, 2002; Roberts et al. 2004; South et al. 2009; Bleu et al. 2016; Foo et al. 2017). Several studies in invertebrates have also demonstrated that there are fitness costs associated with commencing reproduction at an early age (review: Wedell et al. 2002). Many of these studies are, however, correlational. Experiments that measure the costs of mating for individuals that have experienced differences in mating opportunities over significant portions of their reproductive lifespan are needed. But few such studies exist.

Here we test whether sexual experience increases a male's mating success by improving his ability to obtain copulations, despite any associated energetic costs of interacting with females that otherwise reduces investment into costly sexual traits. The eastern mosquitofish, Gambusia holbrooki, is an excellent model system to explore this question (e.g. Bisazza et al. 1996). Males rarely court, and instead pursue and coerce 
118 females into mating. They make frequent attempts to copulate (up to one

119 attempt/minute, Wilson, 2005) by approaching females from behind and thrusting their

120 gonopodium (a modified anal fin used to transfer sperm) towards her gonopore. This

121 makes it less likely that changes in male mating success reflect changes in female

122 mating preferences based on a male's mating history. We maintained male mosquitofish

123 from maturity onward for a prolonged period either with continuous mating access to

124 females ("experienced" males), or in the visual and olfactory presence of females but

125 without physical access to prevent mating ("naïve" males). We predicted that direct

126 sexual experience would benefit males by: (1) improving their ability to approach and

127 inseminate females; (2) increasing their ability to assess which females are more

128 profitable mates (e.g. bigger females who are more fecund, Mautz and Jennions, 2011).

129 But that (3) sexual experience might impose an associated cost since experienced males

130 invested more energy into sperm replenishment and harassment, potentially reducing

131 their ability to invest in other life-history traits such as immune function and growth.

Material and methods

Ethical note

136 The collection of animals was conducted under a Scientific License from the Australian 137 Capital Territory (ACT) Government, granted under Section 21 of the Fisheries Act 2000, license number FS20174. Collection, housing and experimental work conducted as part of the study followed the ASAB/ABS guidelines for the treatment of animals in

140 behavioural research. Information about individuals' housing conditions are described

141 below. Housing conditions, handling and experimental monitoring were conducted so as to maximize the animals' welfare. All experimental procedures were carried out under 


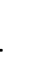

approval from ANU Animal Ethics Committee (Approvals A2015/07 and A2018/27) and complied with existing laws regulating the treatment of vertebrates in Australia.

\section{Origin and maintenance of animals}

In November 2017 we collected immature males $(N=150)$ and recently matured females $(N=300)$ of the mosquitofish $G$. holbrooki from ponds in Australia, where $G$. holbrooki is classified as an invasive pest species. Fish were collected using dip-nets and transported back to the laboratory within $1 \mathrm{~h}$ of collection in groups of 30 individuals in 15 litre containers with portable aeration (size at collection: males $=15$ $20 \mathrm{~mm}$; females 30-45 mm). Males were transferred to individual 1litre tanks, and recently matured females were housed in single-sex 60 litre tanks (50 individuals per tank) containing artificial plants for enrichment and undergravel filter system for aeration. We checked for recovery from capture and transport by confirming that all individuals fed normally within $12 \mathrm{hr}$ of being established in tanks. Fish were maintained in these conditions on a $14: 10 \mathrm{hr}$ light:dark cycle at $27^{\circ} \mathrm{C}( \pm 1)$ for at least two weeks prior to the commencement of the experiment. The actual period depended on the time each male took to reach sexual maturity (range: 2-4 weeks). Experimental males were fed ad libitum with Artemia salina nauplii twice daily, and stimulus females were fed ad libitum twice daily with commercial fish flakes in the morning and $A$. salina nauplii in the afternoon.

Males were checked daily to determine when they reached sexual maturity (i.e. fully formed gonopodium with distal spines). At maturity males were individually transferred to a 4 litre aquarium containing gravel and artificial plants, and randomly assigned to one of two social environment treatments: (1) 'experienced' males had 
access to a female with whom they were able to mate $(N=54)$; (2) 'naïve' males were housed with a female behind a mesh screen to prevent mating, but allow visual contact and the dispersal of chemical cues $(N=51)$. All stimulus females were similar in size (weight $=0.55-0.75 \mathrm{~g}$; 'large' females sensu Head et al. 2015) and were replaced weekly to maintain the males' interest in mating. We assume that 'experienced' males mated regularly with females. This is highly likely because males incessantly attempt to mate (e.g. Wilson, 2005). In this study the artificial plants in the tank provided a partial refuge for the female to mitigate continual male harassment. There was no initial size difference between males in the two treatments $\left(t_{103}=0.093, P=0.355\right)$. After 16 weeks (approximating the typical reproductive lifespan of males in the wild for our study population, Kahn et al. 2013) males were transferred back to individual 1litre tanks for 5d to allow for full sperm replenishment (O’Dea, Jennions, \& Head, 2014). We then ran behavioural assays to test the effect of male mating experience on: (1) the strength of his mating preference for larger females (on average, males prefer larger females; e.g. Mautz and Jennions 2011); (2) his propensity to mate when presented with a female (i.e. chasing behaviour); (3) his actual insemination success. Finally, we measured male immune response with a PHA assay, and recorded his standard length (SL) as a measure of growth (because there was no initial size difference between treatments, this absolute size measure is synonymous with growth). Each behavioural test was conducted by a different observer to prevent biased assessment of the relationship between male performance across tests. All data were collected blind to a male's experience treatment. 

calculated association time with the larger of two females in two-choice trials. The male was introduced to the middle of an aquarium $(49 \times 20 \times 22 \mathrm{~cm})$ containing two end sections of $7 \times 20 \times 22 \mathrm{~cm}$. One section housed a large virgin female $(>45 \mathrm{~mm}$ SL) and the other a small virgin female $(<40 \mathrm{~mm}$ SL). The side containing the larger female was randomly assigned for each trial. Each female was initially behind a mesh divider and an opaque screen. Aquarium walls were lined with black plastic to reduce disturbance. Before the start of the trial, the male was placed in a plastic container in the centre of the test aquarium. After 5 mins of acclimation we carefully removed the container and the opaque screens and then recorded for $10 \mathrm{~min}$ the time the male spent $<5 \mathrm{~cm}$ from the mesh separating him from each female. In some trials $(N=22$ out of 105 total) males did not associate with one of the females. In these cases, we could not be sure that the male had seen ("inspected") both females and therefore that the data represented a preference for one female over the other. We therefore adopted a two-step approach to analyse our male mating preference data (see Statistical Analyses).

Male mating effort

Males were then transferred to a 2 litre tank containing a stock female (mass:

0.55-0.75 g) behind a mesh barrier. All females had originally been collected from the wild, but then housed in single-sex aquaria for $>160$ days to ensure that they were not gravid and were no longer storing sperm from a previous mating (maximum recorded duration of sperm storage is 6 months, Constanz, 1989). The male was given 5 min to acclimate after which the mesh partition was removed and the pair were free to interact. For 10 min we recorded: (1) time spent chasing or associating with the female $(<1$ body length and oriented towards her); (2) number of mating attempts (gonopodial thrusts 
towards her gonopore); (3) number of successful gonopodial thrusts (contacted the gonopore).

\section{Male insemination success}

Males were then left with the female for another $20 \mathrm{~min}$, after which she was removed from the tank, anesthetized in an ice slurry, and her gonoduct flushed with $3 \mu 1$ of saline solution $(0.9 \% \mathrm{NaCl})$ to check for sperm. All females re-attained their swimming equilibrium within 2 minutes of being returned to their tank and behaved normally and feed within minutes of recovery from anaesthesia. There was no mortality.

The sample collected was placed in an Eppendorf tube with another $3 \mu$ of saline solution and vortexed for $30 \mathrm{sec}$ to break up any sperm bundles. We then pipetted $3 \mu 1$ of the vortexed solution onto a 20micron capillary slide (Leja), placed it under a microscope (100 x magnification) and used a CEROS II sperm tracker (Hamilton Thorne Research) to count the number of sperm present for 5 subsamples per slide (repeatability, $r$ : $0.968 \pm 0.012$ s.e). The mean number of sperm was our measure of the relative quantity of sperm transferred.

\section{Male immune response and body size}

Finally, we assessed the cell-mediated immunity of males using a phytohaemagglutinin injection assay (PHA test) to measure their inflammatory response. This assay has been successfully used in other fish (e.g. Clotfelter et al. 2007). We first validated this technique for $G$. holbrooki by comparing the inflammatory response of males injected with either PHA or phosphate-buffered saline (PBS) as a control. There was a clear difference in inflammation between PHA injected males and PBS injected control males, $\left.t_{123}=6.98, P<0.001\right)$. 
We anaesthetized experimental males in an ice slurry then measured the

thickness of the body at the posterior end of the dorsal fin with a pressure-sensitive spessimeter (Mitutoyo 547-301, accuracy: $0.01 \mathrm{~mm}$; average of 5 measurements per fish). We then injected $0.01 \mathrm{mg}$ of PHA dissolved in $0.01 \mathrm{ml}$ of PBS into the right side of the caudal peduncle. Males were returned to their corresponding containers for $24 \mathrm{~h}$, after which we re-measured the thickness of the body at the same point to calculate the difference between pre- and post-injection measures (inflammation). Any tissue swelling provoked by the immune response to the injection of the PHA disappeared after $72 \mathrm{~h}$. All the males behaved normally and fed within half an hour of recovering from the anaesthesia. To control for allometry we recorded male body size (SL, $\pm 0.01 \mathrm{~mm}$ ) using electronic calipers. At the end of the experiment all focal individuals were returned to their 4 litre treatment tanks and after 21 days were euthanised with an overdose of Aqui-S solution (in compliance with Australian government legislation preventing the release of invasive pest species). Non-focal individuals were returned to stocking tanks to be used, where possible, in subsequent experiments.

Statistical analyses

We analysed data using separate Generalized Linear Models (GLM), with the choice of error function for each model based on inspection of the distribution of dependent variable and confirmed via Q-Q plots of model residuals. When testing for the effect of mating experience on a preference for large versus small females, we tested first for the effect of experience on the propensity to inspect both females (GLM, 
male mating experience on: the proportion of time spent with the large female

268 (Quasibinomial error, cbind function $c f$ Deere et al. 2012; Booksmythe et al. 2013;

Vega-Trejo et al. 2014; Wacker et al. 2016). We ran separate GLMs to test for the effect of male experience on: time spent chasing the female (Quasipoisson error), number of gonopodial thrusts (Quasipoisson error), the proportion of successful gonopodial thrusts

272 (Quasibinomial error, cbind function), whether or not a male transferred sperm

273 (Quasibinomial error), the quantity of sperm transferred by the 23 "naïve" and 19 "experienced" males who successfully inseminated a female (Gaussian error), immune response (Gaussian error) and growth over the treatment period (i.e. final size) (Gaussian error). Sperm quantity and immune responses were log-transformed to meet assumptions of normality. In all models male mating experience (naïve or experienced) was treated as a fixed factor.

Since male body size might be an important factor driving the behavioural trials we initially ran models including male size as covariate. Including this variable did not affect any of the results, so for simplicity we report models without the covariate. Body size was, however, included as a covariate in the immune response model as it was correlated with the amount of post-injection inflammation $(r=0.28, P=0.004)$. We excluded one outlier from the immune response model because it had an extreme value that is likely to reflect measurement error or a mistake in data transcription. The mean inflammation value was $0.174 \pm 0.114$ s.d. $(N=105$ males $)$, while the outlier had a value of 0.572 (i.e. > 3.5 standard deviations from the mean). However, to ensure transparency we also report model results based on the full dataset. 
Results

295

When males were given a choice between two females, experienced males were

297

298

299

300

301

302

303

304

305

306

307

308

309

310

311

312

313

314

315

316 significantly more likely to inspect both females $\left(\chi^{2} 104=6.035, P=0.014\right)$. Of those males that inspected both females, experienced males spent significantly more time than naïve males in association with the larger of the two females $\left(t_{81}=2.628, P=0.010\right.$, Fig 1a). For naïve males, there was no difference in the proportion of time spent with the large and small female $\left(t_{34}=0.221, P=0.826\right)$. However, a male's previous mating experience did not affect his mating effort. There was no difference between experienced and naïve males in the time spent chasing a female $\left(t_{103}=0.135, P=0.893\right.$, Fig $1 b)$, in the number of mating attempts $\left(t_{103}=1.332, P=0.186\right.$, Fig $\left.1 \mathrm{c}\right)$, in the likelihood of transferring sperm $\left(t_{103}=1.024, P=0.308\right.$, Fig $\left.1 \mathrm{~d}\right)$, or, if insemination occurred, the quantity of sperm transferred $\left(t_{40}=0.642, P=0.524\right)$. Interestingly, however, the proportion of successful gonopodial thrusts made by naïve males was marginally non-significantly greater than that of experienced males $\left(t_{103}=1.886, P=\right.$ $0.059)$.

The opportunity to mate with females had a negative effect on post-maturation growth, such that experienced males were significantly smaller in size than naïve males $\left(t_{104}=3.857, P<0.001\right)$ (Fig $\left.2 \mathrm{a}\right)$. In addition, experienced males had a significantly weaker immune response than naïve ones $\left(t_{101}=2.049, P=0.043\right)$. However, if we included an outlier in our model there was no longer a significant effect of the experience treatment on male immune response $\left(t_{102}=1.647, P=0.103\right.$, Fig $\left.2 b\right)$. 
Discussion

320

By manipulating the access of male $G$. holbrooki to females over their entire

322

323

324

325

326

327

adult lifespan prior to testing, we explored whether direct sexual experience increased male mating success due to learning how to be more effective at gaining copulations. More specifically, we tested how adult sexual experience affected a male's ability to mate, independent of female mating preferences for males with different mating histories. This claim is based on the assumption that female choice for more experienced males is unlikely due to the coercive mating system. The use of adults in our experiment contrasts with an earlier study in G. holbrooki that explored the effect of exposure to females when males were still juveniles on their subsequent adult sexual behaviour (Bisazza et al 1996). We tested whether sexual experience increased a male's reproductive success in terms of both his ability to gain copulations and his ability to discriminate between potential mates based on their size. We predicted that if interacting with females allows males to learn, then sexually experienced male $G$. holbrooki would be more likely to achieve coercive copulations, and would show stronger preferences for more fecund mates (i.e. larger females). Our prediction about female choice is based on earlier work showing that males are more likely to copulate when they attempt to mate with larger females (Pilastro, Giacomello \& Bisazza, 1997). Males should therefore learn that larger females are more profitable, at least in terms of the likelihood of mating (but see Fitzpatrick \& Servedio, 2018 who review the evidence that more fecund females are 'devalued' due to an increase in shared paternity if males 
341 preferentially mate with them). We also sought to confirm that sexually experienced

342 males pay a cost due to their greater past mating effort.

As predicted, experienced males differed from naïve males in their mate preferences, spending significantly more time associating with the larger of two females. Experienced males also grew more slowly and had a weaker immune response than naïve males, confirming that mating effort is costly. However, contrary to our predictions, experienced males did not make significantly more copulations attempts than naïve males, nor were they more likely to inseminate females. Intriguingly, the proportion of successful gonopodial thrusts made by naïve males was marginally nonsignificantly greater than that of experienced males. There is therefore no evidence that experienced males with the opportunity to interact with females over their adult lifespan had an advantage over naïve males because they learned to mate more successfully. recognition (e.g., Svensson et al. 2010), or how social cues influence mating decisions (via mate-choice copying) (e.g., Schlupp \& Ryan, 1996; Widemo, 2006; Witte \& Ryan, 2002; review: Schlupp, 2018). Fewer studies have examined the role of learning to discriminate between conspecific mates based on their quality, and/or the likelihood of obtaining a copulation. Mating is generally costly for males due to sperm and energy expenditure, so when the costs of choice are low, as is always the case when two

362 females are simultaneously available (Barry and Kokko, 2010), males are expected to direct their mating efforts towards high quality females (Bonduriansky, 2001). In our study, experienced males had a stronger preference for associating with larger, more fecund, females, and, hence, a stronger ability to discriminate between partners based on 
their quality under low-cost conditions (but see Fitzpatrick \& Servedio, 2018 for the potentially confounding effect of greater shared paternity). There are several potential explanations for this result. First, the difference between experienced and naïve males in time spent in association with larger females might be related to learning about variation in actual female quality (Verzijden et al. 2012). However, this would require males to have had previous access to females of different phenotypes and being able to ascertain the number and quality of offspring they sired. This is implausible. Second, experienced males might have learnt that they are more successful at copulating with larger females as a greater size difference between males and females increases a male's mating success (see Bisazza \& Marin, 1995; Pilastro et al. 1997). Third, weaker discrimination by naïve males could be a strategy to reduce lost mating opportunities at the end of their reproductive lifespan by minimizing the time spent searching for, or inspecting, females. Similarly, the fact that naive males were less likely to "inspect" both females could mean that "inspection" is learned.

Contrary to findings in some species (e.g. Dukas, 2005; Edvardsson et al. 2008;

Saleem et al 2014), sexual experience did not improve male G. holbrooki mating ability, when measured as: (1) the number of gonopodial thrusts; (2) the proportion of successful thrusts; (3) the rate of successful sperm transfer; (4) the amount of sperm transferred. Although mating in G. holbrooki appears to result from male coercion, there is some evidence that female choice can still generate mating biases because females are more likely to associate with, hence be harassed by, certain males (Bisazza, Vaccari, \& Pilastro, 2001; Kahn, Livingston, \& Jennions, 2012; Kahn, Mautz, \& Jennions, 2010). As such, it is still possible that sexual experience is beneficial to male G. holbrooki if it increases the likelihood that females associate with experienced males (but see Jiménez- 
Pérez and Wang, 2004; King and Fischer, 2010). However, to detect this, it will be necessary to carry out female mate choice trials in which the effects of male age and experience are teased apart.

Sexual experience did not increase the likelihood of successful sperm transfer or the amount of sperm transferred when males copulated. We expected naïve males, who had never mated, to invest more into sperm production due to their perception that mating opportunities are rare (see earlier discussion above indiscrimination mate choice being due to perceived high opportunity costs). All else being equal, differences in previous opportunities to mate should promote production of a larger ejaculate based on the information that there will be enough time for sperm replenishment before encountering another female (Parker \& Pizzari, 2010; Wedell et al. 2002). Our results suggest that, despite the extreme difference in prior mating opportunities, experienced and naïve males produced the same size ejaculates.

In $G$. holbrooki there was no effect of sexual experience on the time males spent chasing a female, or on the rate of copulation attempts. This contrasts with other species where past encounters with females affect male mating effort (Balaban-Feld \& Valone, 2017; Saleem et al. 2014). For example, in fruitflies, experienced males were less likely to behave aggressively and to use coercion against females than were naïve males (Baxter \& Dukas, 2017). Alternatively, naïve males might have behaved more aggressively because they perceived their future reproduction opportunities to be low (Fischer et al., 2008) as, despite exposure to females, they had previously failed to mate. For example, Bisazza et al (1996) raised juvenile male G. holbrooki in the presence or absence of females and found that, upon adulthood, naïve males made significantly 
more copulatory attempts, and a greater absolute number of successful copulatory attempts than experienced males, but copulation efficiency was broadly equivalent for naïve and experienced males. In our study, naïve and experienced males did not differ in the number of copulation attempts, but the proportion of successful attempts by naïve males was marginally non-significantly greater than that of experienced males $(\mathrm{P}=$ 0.059). There are many untestable reasons why the results of our study and that of Bisazza et al (1996) might differ (e.g. population differences, test conditions, sampling error). The most obvious difference is, however, whether males were exposed to females to gain experience as juveniles (Bisazza et al. 1996) or adults (our study). Nonetheless, both studies suggest that male G. holbrooki do not significantly improve the effectiveness of their coercive behaviour by having the opportunity to interact with females (i.e. learn). their greater mating effort, experienced male G. holbrooki grew more slowly than naïve males and tended to have a weaker immune response. Our results suggest that continuous harassment of females and sperm replenishment are therefore costly in species with coercive mating tactics. Studies of other poecilids have similarly shown that mating effort can reduce male growth (Jordan \& Brooks, 2010). Our finding of a reduced immune response in experienced males is consistent with the idea that mating effort suppresses immunity (Folstad \& Karter, 1992). This has often been demonstrated in invertebrates (e.g., McKean \& Nunney 2001; Rolff \& Siva-Jothy 2002; Fedorka et al. 2004; review: Lawniczak et al. 2007), and in some vertebrates (e.g. birds: Roberts et al. 2004; mammals: Muehlenbein \& Bribiescas, 2005). 
Past sexual experience did not confer an advantage to male G. holbrooki by improving

their ability to inseminate a female. This suggests that having the opportunity to interact

with females did not cause males to learn how to be more effective at completing

copulations and transferring sperm. There was, however, a potential benefit of interacting with females if the mate choice experiment is interpreted as evidence that sexually experienced males improved their ability to direct their mating effort towards large more fecund females. However, this type of male preferences might be disadvantageous (or confer no advantage) if males more often copulate with preferred, large females, thereby reducing the mean paternity per male (Servedio, 2007; Fitzptarick \& Servedio, 2018). In addition, we confirmed there are significant costs of mating effort for males over their reproductive lifespan. Future studies examining the effects of lower immunity and slower growth of male lifetime reproductive success would be useful to further our understanding of life-history trade-offs (Brooks \& Garratt, 2017).

\section{Author contributions}

MLH and MDJ conceived the study, MLH, RJF and MIC designed the study, MIC, RJF and AV collected the data, MIC and RJF analysed the data. All authors interpreted the data, co-wrote the manuscript and gave permission for publication.

\section{Competing interests}

465 The authors declare no competing interests. 
Data availability

468

Data will be made available on request.

469

\section{Acknowledgements}

471 We thank Regina Vega-Trejo, Upama Aich, Milan Vrtilek and ANU Animal Services

472 for assistance with fish husbandry. This research was funded by the Australian

473

Research Council (DP160100285 awarded to MDJ and FT160100149 awarded to

474 MLH).

475

476

References

477

478

Balaban-Feld, J., \& Valone, T. J. (2017). Prior information and social experience

479

influence male reproductive decisions. Behavioral Ecology, 28, 1376-1383.

480

Barnes, A., \& Partridge, L. (2004). Costing reproduction. Animal Behaviour, 66, 199-

481 204.

482

Barry, K. L., \& Kokko, H. (2010). Male mate choice: Why sequential choice can make its evolution difficult. Animal Behaviour, 80, 163-169.

484

Baxter, C. M., \& Dukas, R. (2017). Life history of aggression: effects of age and sexual experience on male aggression towards males and females. Animal Behaviour, 123, 1120.

487 Bisazza, A., \& Marin, G. (1995). Sexual selection and sexual size dimorphism in the 488 eastern mosquitofish Gambusia holbrooki (Pisces Poeciliidae). Ethology, Ecology \& 489 Evolution, 7, 169-183.

Bisazza, A., Pliastro, A., Palazzi, R., \& Marin G. (1996). Sexual behaviour of immature 
491

492

493

494

495

496

497

498

499

500

501

502

503

504

505

506

507

508

509

510

511

512

513

514

515

male eastern mosquitofish: a way to measure intensity of intra-sexual selection? Journal of Fish Biology, 48, 726-737.

Bisazza, A., Vaccari, G., Pilastro, A. (2001). Female mate choice in a mating system dominated by male sexual coercion. Behavioral Ecology, 12, 59-64.

Bleu, J., Gamelon, M., \& Sæther, B. E. (2016). Reproductive costs in terrestrial male vertebrates: Insights from bird studies. Proceedings of the Royal Society of London. Series B, Biological Sciences, 283, 20152600.

Bonduriansky, R. (2001). The evolution of male mate choice in insects : a synthesis of ideas and evidence. Biological Reviews, 76, 305-339.

Bonduriansky, R. (2007). The evolution of condition dependent sexual dimorphism. American. Naturalist, 169, :9-19.

Booksmythe, I., Backwell, P. R. Y., \& Jennions, M. D. (2013). Competitor size, male mating success and mate choice in eastern mosquitofish (Gambusia holbrooki). Animal Behaviour, 85, 371-375.

Bretman, A., Gage, M. J. G., \& Chapman, T. (2011). Quick-change artists: Male plastic behavioural responses to rivals. Trends in Ecology \& Evolution, 26, 467-473.

Brooks, R., \& Kemp, D. J. (2001). Can older males deliver the good genes? Trends in Ecology \& Evolution, 16, 308-313.

Brooks, R. C., \& Garratt, M. G. (2017). Life history evolution, reproduction, and the origins of sex-dependent aging and longevity. Annals of the New York Academy of Sciences, 1389, 92-107.

Brown, G., \& Fawcett, T. (2005). Sexual selection: copycat mating in birds. Current Biology, 15, 627-628.

Callander, S., Backwell, P. R. Y., \& Jennions, M. D. (2012). Context-dependent male mate choice: The effects of competitor presence and competitor size. Behavioral 
517 Clotfelter, E. D., Ardia, D. R., \& McGraw, K. J. (2007). Red fish, blue fish: Trade-offs 518 between pigmentation and immunity in Betta splendens. Behavioral Ecology, 18, 11395191145.

520 Constanz, G. (1989). Reproductive biology of peociliid fishes. In: G. Meffe \& F.

521 Snelson (Eds.), Ecology and Evolution of Live Bearing Fishes (Poeciliidae). (pp. 149522 161). New Jersey, Prentice Hall.

523 Deere, K. A., Grether, G. F., Sun, A., \& Sinsheimer J. S. (2012). Female mate 524 preference explains countergradient variation in the sexual coloration of guppies 525 (Poecilia reticulata). Proceedings of the Royal Society of London. Series B, Biological 526 Sciences, 279, 1684-1690.

527 Dukas, R. (2005). Experience improves courtship in male fruit flies. Animal Behaviour, 528 69, 1203-1209.

Dukas, R., Clark, C. W., \& Abbott, K. (2006). Courtship strategies of male insects:

530 when is learning advantageous? Animal Behaviour, 72, 1395-1404.

531 Edvardsson, M., Hunt, J., Moore, P. J., \& Moore, A. J. (2008). Female agreement over male attractiveness is not affected by cost of mating with experienced males. Behavioral Ecology, 19, 854-859.

534 Fedorka, K. M., Zuk, M., \& Mousseau, T. A. (2004). Immune suppression and the cost 535 of reproduction in the ground cricket, Allonemobius socius. Evolution, 58, 2478-2485. 536 Fincke, O. M., Fargevieille, A., \& Schultz, T. D. (2007). Lack of innate preference for 537 morph and species identity in mate-searching Enallagma damselflies. Behavioral 538 Ecology and Sociobiology, 61, 1121-1131.

539 Fischer, K., Perlick, J., \& Galetz, T. (2008). Residual reproductive value and male 540 mating success: older males do better. Proceedings of the Royal Society of London. 
542 Fitzpatrick, J. L., \& Lüpold, S. (2014). Sexual selection and the evolution of sperm quality. Molecular Human Reproduction, 20, 1180-1189.

544 Fitzpatrick, C.L. \& Servedio, M.R. (2018) The evolution of male mate choice and 545 female ornamentation: a review of mathemetaical models. Current Zoology 64, 323546333.

547 Folstad, I., \& Karter, A. J. (1992). Parasites, bright males and the immunocompetance handicap. American Naturalist, 139, 603-622.

549 Foo, Y. Z., Nakagawa, S., Rhodes, G., \& Simmons, L.W. (2017). The effects of sex 550 hormones on immune function: a meta-analysis. Biological Reviews, 92, 551-571.

551 Gasparini, C., Serena, G., \& Pilastro, A. (2013). Do unattractive friends make you look better? Context-dependent male mating preferences in the guppy. Proceedings of the Royal Society B: Biological Sciences, 280, 20123072. females mate choice in the guppy, Poecilia reticulata: generalised and repeatable traitcopying behaviour. Animal Behaviour, 69, 999-1005.

557 Head, M.L., Vega Trejo, R., Jacomb, F., \& Jennions, M.D. (2015). Predictors of male 558 insemination success in the mosquitofish (Gambusia holbrooki). Ecology and Evolution 5, 4999-5006

560 Immelmann, K. (1975). Ecological significance of imprinting and early learning. 561 Annual Review of Ecology and Systematics, 6, 15-37.

562 Jiménez-Pérez, A. A., \& Wang, Q. (2004). Sexual selection in Cnephasia jactatana 563 (Lepidoptera: Tortricidae) in relation to age, virginity, and body size. Annals of the 564 Entomological Society of America, 97, 819-824.

565 Jordan, L. A., \& Brooks, R. C. (2010). The lifetime costs of increased male 
566

567

568

569

570

571

572

573

574

575

576

577

578

579

580

581

582

583

584

585

586

587

588

589

590

reproductive effort: Courtship, copulation and the Coolidge effect. Journal of Evolutionary Biology, 23, 2403-2409.

Kahn, A. T., Kokko, H., \& Jennions, M. D. (2013). Adaptive sex allocation in anticipation of changes in offspring mating opportunities. Nature Communications, 4 , 1603-1607.

Kahn, A. T., Livingston, J. D., \& Jennions, M. D. (2012). Do females preferentially associate with males given a better start in life? Biology Letters, 8, 362-364.

Kahn, A. T., Mautz, B., \& Jennions, M. D. (2010). Females prefer to associate with males with longer intromittent organs in mosquitofish. Biology Letters, 6, 55-58.

Kelly, C. D., \& Godin, J. G. J. (2001). Predation risk reduces male-male sexual competition in the Trinidadian guppy (Poecilia reticulata). Behavioral Ecology \& Sociobiology, 51, 95-100.

Kelly, C. D., \& Jennions, M. D. (2011). Sexual selection and sperm quantity: Metaanalyses of strategic ejaculation. Biological Reviews, 86, 863-884.

King, B. H., \& Fischer, C. R. (2010). Male mating history: Effects on female sexual responsiveness and reproductive success in the parasitoid wasp Spalangia endius. Behavioral Ecology \& Sociobiology, 64, 607-615.

Kolluru, G. R., Grether, G. F. (2005). The effects of resource availability on alternative mating tactics in guppies (Poecilia reticulata). Behavioral Ecology, 16, 294-300.

Koref-Santibáñez, S. (2001). Effects of age and experience on mating activity in the sibling species Drosophila pavani and Drosophila gaucha. Behavior Genetics, 31, 287297.

Kotiaho, J. S. (2001). Costs of sexual traits: a mismatch between theoretical considerations and empirical evidence. Biological Reviews, 76, 365-376.

Kuijper, B., Pen, I., \& Weissing, F. J. (2012). A guide to sexual selection theory. 
591

592

593

594

595

596

597

598

599

600

601

602

603

604

605

606

607

608

609

610

611

612

613

614

615

Annual Review of Ecology, Evolution \& Systematics, 43, 287-311.

Lawniczak, M. K. N., Barnes, A. I., Linklater, J. R., Boone, J. M., Wigby, S., \&

Chapman, T. (2007). Mating and immunity in invertebrates. Trends in Ecology and Evolution, 22, 48-55.

Macario, A., Croft, D. P., Endler, J. A., \& Darden, S. K. (2017). Early social experience shapes female mate choice in guppies. Behavioral Ecology, 28, 833-843.

Mautz, B. S., \& Jennions, M. D. (2011). The effect of competitor presence and relative competitive ability on male mate choice. Behavioral Ecology, 22, 769-775.

McKean, K. A., \& Nunney, L. (2001). Increased sexual activity reduces male immune function in Drosophila melanogaster. Proceedings of the National Academy of Sciences of the United States of America, 98, 7904-7909.

Mery, F., \& Kawecki, TJ. 2003. A fitness cost of learning ability in Drosophila melanogaster. Proceedings of the Royal Society of London. Series B, Biological Sciences 270, 2465-2469.

Muehlenbein, M. P., \& Bribiescas, R. G. (2005). Testosterone-mediated immune functions and male life histories. American Journal of Human Biology, 17, 527-558.

O’Dea, R. E., Jennions, M. D, \& Head, M. L. (2014). Male body size and condition affects sperm number and production rates in mosquitofish, Gambusia holbrooki. Journal of Evolutionary Biology, 27, 2739-2744.

Olsson, M., Madsen, T., \& Shine, R. (1997). Is sperm really so cheap? Costs of reproduction in male adders, Vipera berus. Proceedings of the Royal Society of London. Series B, Biological Sciences, 264, 455-459.

Parker, G. A., \& Pizzari, T. (2010). Sperm competition and ejaculate economics. Biological Reviews, 85, 897-934.

Pérez-Staples, D., Martínez-Hernández, M. G., \& Aluja, M. (2010). Male age and 
experience increases mating success but not female fitness in the mexican fruit fly.

617 Ethology, 116, 778-786.

618 Pilastro, A., Giacomello, E., \& Bisazza, A. (1997). Sexual selection for small size in male mosquitofish (Gambusia holbrooki). Proceedings of the Royal Society of London. Series B, Biological Sciences, 264, 1125-1129.

Reif, M., Linsenmair, K. E., \& Heisenberg, M. (2002). Evolutionary significance of courtship conditioning in Drosophila melanogaster. Animal Behaviour, 63, 143-155. immunocompetence handicap hypothesis: A review of the evidence. Animal Behaviour, $68,227-239$.

Rolff, J., \& Siva-Jothy, M. T. 2002. Copulation corrupts immunity: A mechanism for a cost of mating in insects. Proceedings of the National Academy of Sciences of the United States of America, 99, 9916-9918. enhances Drosophila melanogaster male mating behavior and success. PLoS ONE, 9, e 96639.

Schlupp, I. (2018). Male mate choice in livebearing fishes: an overview. Current Zoology 64, 393-403.

634 Schlupp, I., \& Ryan, M. (1996). Male sailfin mollies (Poecilia latipinna) copy the mate choice of other males. Behavioral Ecology, 8, 104-107.

Servedio, M. R. (2007). Male versus female mate choice: Sexual selection and the evolution of species recognition via reinforcement. Evolution, 61, 2772-2789. 
641 Svensson, E. I., Eroukhmanoff, F., Karlsson, K., Runemark, A., \& Brodin, A. (2010). A

642 role for learning in population divergence of mate preferences. Evolution, 64, 3101-

$643 \quad 3113$

644 Swaddle, J. P., Cathey, M. G., Correll, M., \& Hodkinson BP. 2005. Socially transmitted 645 mate preferences in a monogamous bird: a non-genetic mechanism of sexual selection. 646 Proceedings of the Royal Society of London. Series B, Biological Sciences 272:1053$647 \quad 1058$.

648 Vega-Trejo, R., O'Dea, R. E., Jennions, M. D., \& Head, M. L. (2014). The effects of 649 familiarity and mating experience on mate choice in mosquitofish, Gambusia holbrooki. 650 Behavioral Ecology, 25, 1205-1211.

651 Verzijden, M. N., ten Cate, C., Servedio, M. R., Kozak, G. M., Boughman, J. W., \& 652 Svensson, E. I. (2012). The impact of learning on sexual selection and speciation. 653 Trends in Ecology \& Evolution, 27, 511-519.

654 Wacker, S., Östlund-Nilsson, S., Forsgren E., Newport, C., \& Amundsen T. (2016).

655 Mate choice plasticity in a coral reef fish. Behavioral Ecology, 27, 1331-1342.

656 Wedell, N., Gage, M. J. G., \& Parker, G. A. (2002). Sperm competition, male prudence, 657 and sperm-limited females. Trends in Ecology \& Evolution, 17, 47-63.

658 Wedell, N., \& Ritchie, M. G. (2004). Male age, mating status and nuptial gift quality in 659 a bushcricket. Animal Behaviour, 67, 1059-1065.

660 Widemo, M. S. (2006). Male but not female pipefish copy mate choice. Behavioral 661 Ecology, 17, 255-259.

662 Wilson, R. S. (2005). Temperature influences the coercive mating and swimming 663 performance of male eastern mosquitofish. Animal Behaviour, 70, 1387-1394.

664 Witte, K., \& Ryan, M. J. (2002). Mate choice copying in the sailfin molly, Poecilia 665 latipinna, in the wild. Animal Behaviour, 63, 943-949. 
666 Figure 1. The effect of previous sexual experience on (a) the time spent associating with

667 the larger of two females in two-choice trials beyond that expected due to chance alone

668 (i.e. if males spent half of each trial (300s) with each female). The 'extra time' is

669 therefore the actual time minus 300s; (b) time spent chasing the female; (c) number of

670 mating attempts; (d) sperm transfer success. (Mean \pm s.e).

671

672 Figure 2. Effect of previous sexual experience on (a) male body size and (b) immune

673 response (raw data are shown). The red point in (b) represents a male with an extreme

674 immune response value. There is also one male with a large body size $(>24 \mathrm{~mm})$ in the

675 naïve group (panel (a)), but excluding this male does not change the main result that

676 access to females reduces male growth (see text) (excluded: $t_{102}=3.696, P<0.001$ ). 

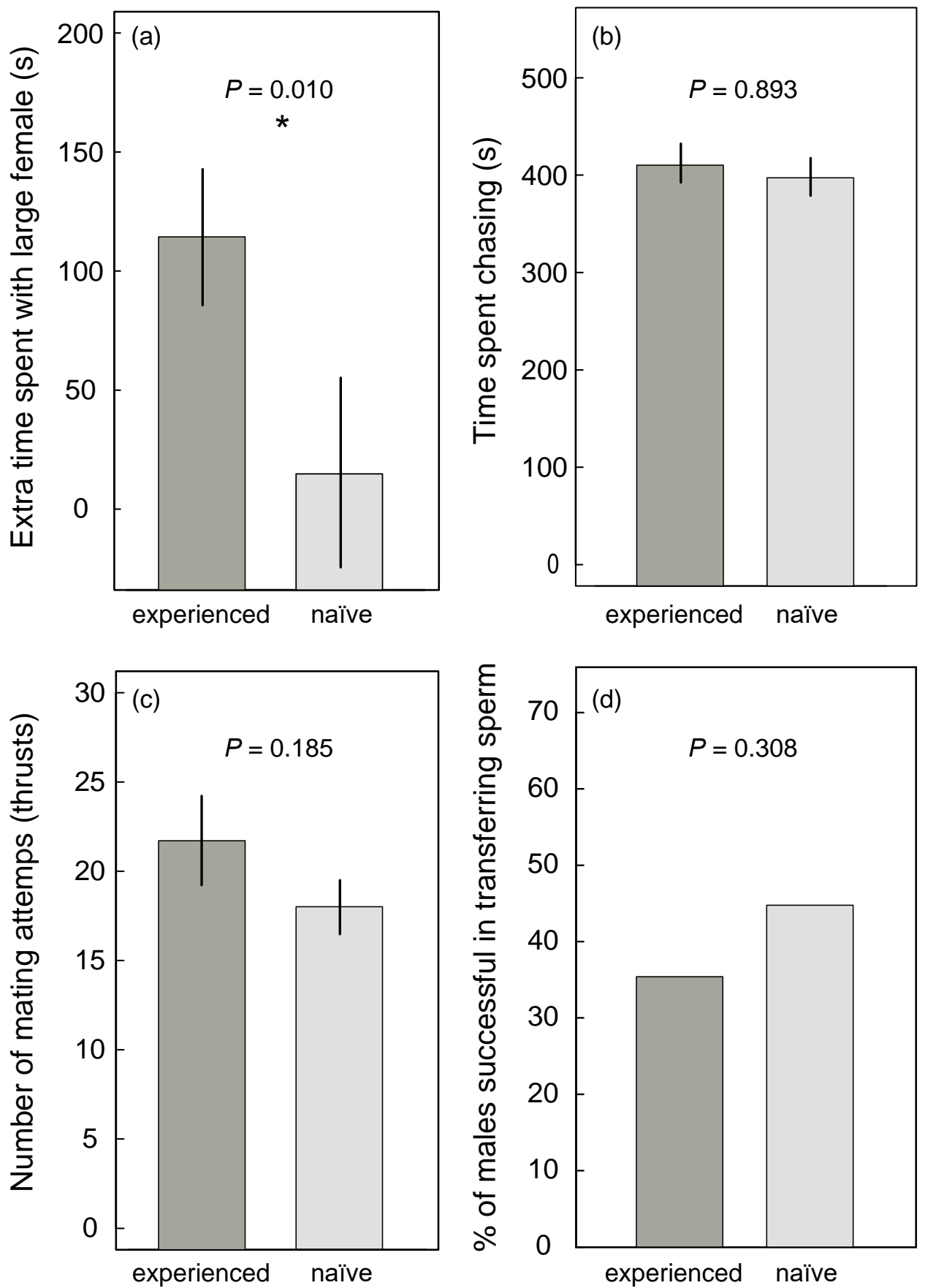
Fig. 2
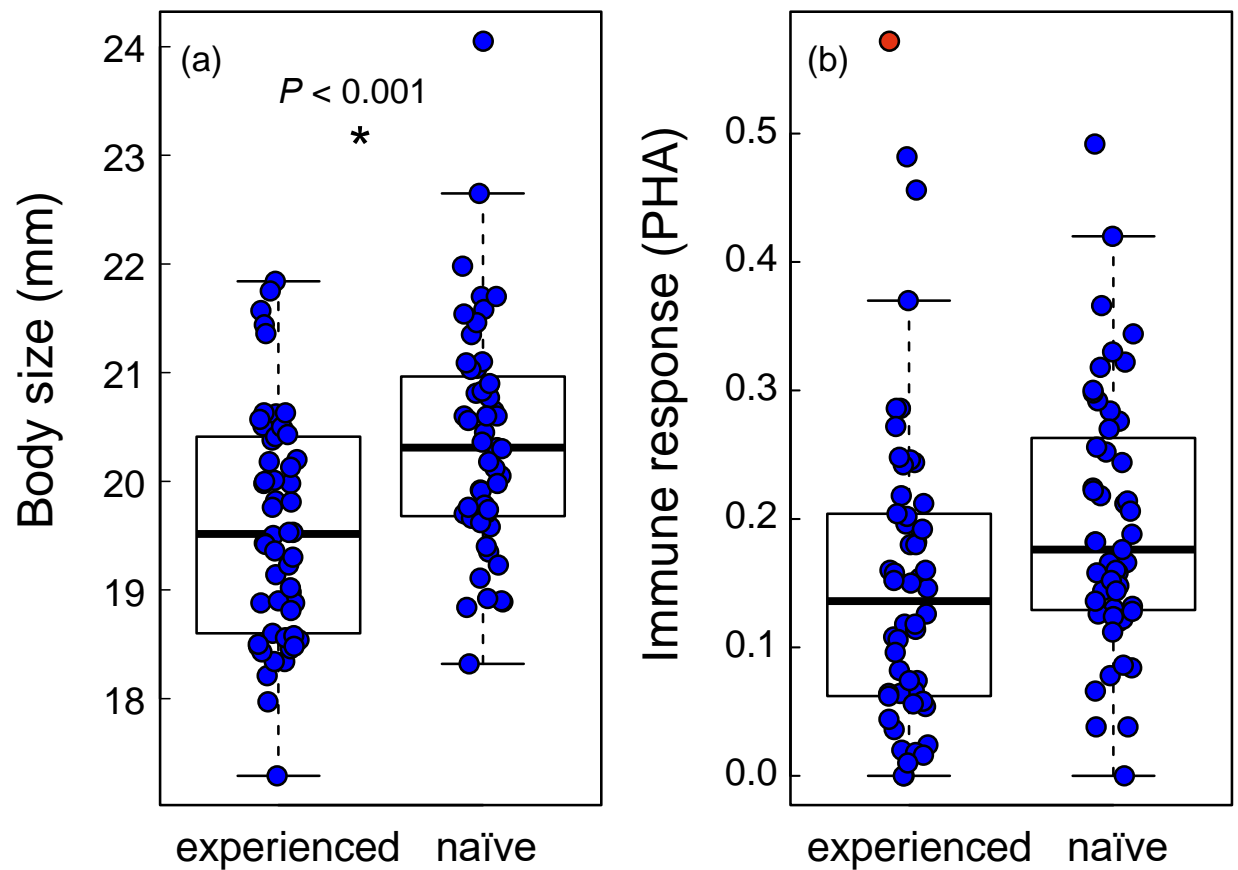

Treatment 